\title{
A SUPERCONDUCTING SOLENOID FOR HEAVY ION BEAM FOCUSING
}

\author{
J.W. Kim, T. Kubo, T. Kawaguchi, \\ RIKEN, Saitama, Wako-shi, 351-01 Japan \\ Y. Imai, T. Minato, and K. Seo, \\ Mitsubishi Electric Corporation, Wadasaki-cho, Hyogo-ku, Kobe, 661 Japan
}

\begin{abstract}
A 6 T superconducting solenoid has been constructed to focus heavy ion beams on the production target of radio isotope beams. The design field on axis is $6.2 \mathrm{~T}$ with an average current density of $9,600 \mathrm{~A} / \mathrm{cm}^{2}$. The overall coil length is 1.1 meter, and the coil is divided into three equal-length sections to ease winding and to reduce IR drop through diode connection. A main feature of the system is that the solenoid is conductively cooled by a cryocooler directly attached onto the center coil without liquid helium involved. The solenoid was successfully trained to $6.2 \mathrm{~T}$, and its optical properties were tested in the beamline at RIKEN. The design features and the test results are presented.
\end{abstract}

\section{INTRODUCTION}

The accelerator research facility at the Institute of Physical and Chemical Research (RIKEN) in Japan has provided high current radio isotope beams for nuclear physics research [1]. To produce isotope beams a projectile fragmentation method is being used. By bombarding a production target with heavy ion beam, fragments of the projectile are produced. The Radio Isotope Projectile Separator (RIPS) located downstream then selects the desired beam [2].

Until recently the primary beam has been focused on target using quadrupole lens, the resulting beam spot being larger than $1.5 \mathrm{~mm}$, which meets usual experimental requirements. However, a need for higher precision measurements has demanded even smaller beam spot. After some computational studies and comparison works, a superconducting solenoid was chosen to increase the focusing power with a slight modification of the existing beamline. A quadrupole triplet was also an option, but the solenoid appeared to be more attractive in the aspect of its versatile uses. One example of its uses is that with a production target placed upstream from the solenoid, the solenoid can accept and focus the produced fragments on another production target downstream.

The solenoid was manufactured by Mitsubishi Electric Corporation, and was installed in the beamline at RIKEN in January, 1997. The axial field reached 6.2 T after several quenches. The first focusing test was performed using $95 \mathrm{MeV} / \mathrm{u} \mathrm{Ar}^{17+}$ beam, which is the highest rigidity beam from RIKEN Ring Cyclotron (RRC).

\section{SUPERCONDUCTING SOLENOID DESIGN}

The superconducting solenoid is designed as a focusing element in the beamline. Since no present beamline mag- nets at RIKEN are superconducting, it was thought to be inconvenient to add a new component which requires using liquid helium. A conduction cooling method was preferred to bath cooling. A compact refrigerator of a GiffordMcMahon type and a high- $\mathrm{T}_{c}$ material current leads are the major components of such system as in other similar systems [3]. (The current leads is made of high- $\mathrm{T}_{c}$ material between $50 \mathrm{~K}$ and $4.5 \mathrm{~K}$, and of copper between $300 \mathrm{~K}$ and 50 K.) Figure 1 shows the system configuration. Design parameters of the solenoid and the superconductor are listed in Table 1.

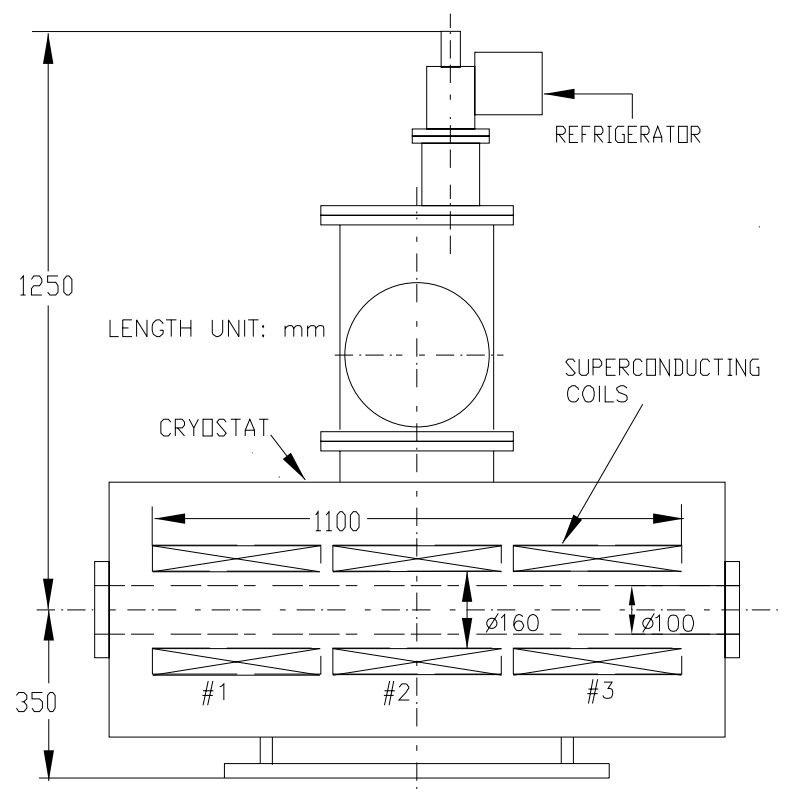

Figure 1: A schematic view of the superconducting solenoid.

The solenoid is divided into three equal-length coils as shown in Fig. 1. Its main purpose is to reduce the maximum IR drop. Each coil is connected to a diode so that the maximum IR drop during quench is reduced approximately by one third compared to without coil division. The electric circuit incorporating quench protection is plotted in Figure 2. Three diodes are located inside the cryostat, and there is another diode used at room temperature in series with a resistor which are in a parallel connection with the power supply. Because of these connections quench characteristics are rather complicated. A detailed analysis is given in the next section.

The voltage of power supply is \pm 4 volts, taking about 90 mins to reach the design current. It is, however, not a 
Table 1: Parameters of the 6 T Solenoid

\begin{tabular}{|c|c|}
\hline $\mathrm{B}_{\max }$ on axis & $6.2 \mathrm{~T}$ \\
\hline $\int B \cdot d l$ & $6.8 \mathrm{~T} \cdot \mathrm{m}$ \\
\hline $\mathrm{J}_{\text {ave }}$ & $9,800 \mathrm{~A} / \mathrm{cm}^{2}$ \\
\hline Amp turn & $5.5 \mathrm{MA} \cdot \operatorname{turn}$ \\
\hline $\mathrm{I}_{o p}$ & $40 \mathrm{~A}$ \\
\hline Stored Energy & $450 \mathrm{~kJ}$ \\
\hline Inductance & $534 \mathrm{H}$ \\
\hline $\begin{array}{c}\text { Superconducting } \\
\text { Wire }\end{array}$ & $\begin{array}{c}\mathrm{Cu} / \mathrm{NbTi}=1.3, \\
\phi=0.60 \mathrm{~mm}\end{array}$ \\
\hline
\end{tabular}

significant inconvenience in our case because the current is usually fixed for many hours during experiments.

The cold mass of the solenoid is about $300 \mathrm{~kg}$, and the cooling capacity of the refrigerator is $0.5 \mathrm{~W}$ at $4.5 \mathrm{~K}$ with $30 \mathrm{~W}$ being provided to the thermal shield at $45 \mathrm{~K}$. It takes 9 days to cool the coil from $300 \mathrm{~K}$ to $4.5 \mathrm{~K}$. This seems a rather long time, but is acceptable since experiments are usually scheduled well in advance.

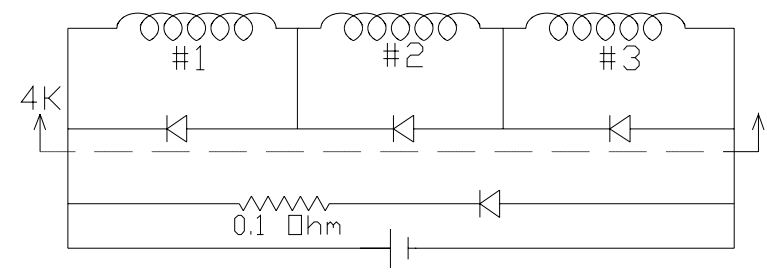

Figure 2: A circuit diagram. Three diodes are located inside the cryostat, and one more diode at room temperature for quench protection.

\section{SOLENOID TEST AND FIELD MEASUREMENT}

The solenoid reached the design field of 6.2 tesla after training. Among the three coils, two (\#1 and \#3 in Figure 1) were tested in a liquid helium dewar prior to assembly. The coil \#1 quenched at the current of $48.3 \mathrm{~A}$, and the coil \#3 didn't even at 49.1 A, which proved the quality of coil winding. Test of the third coil was skipped. After the solenoid was fully assembled, it was first tested at the factory site. And then the test continued after the solenoid was installed in the beamline of RIKEN. Twelve quenches in total were needed to train the solenoid to the design field. This number is larger than expected. The quench occured at the coil \#3 for the first 4 quenches, and then at \#1 for the following 7 quenches. Since these two coils passed individual tests, the quality of winding should not be the cause of these quenches. Moreover, the coil joints are extra-cooled by means of separate copper strips extended from the cold head of the refrigerator. We think that the change of coil force direction from individual coil tests is the probable cause.
During quench tests variations of the following physical parameters have been measured: 1) voltages on each coil, 2) magnetic fields, and 3) solenoid current. The magnetic fields were measured using three hall probes temporarily attached to the outside of vacuum jacket around each coil. A typical result is shown in Figure 3 when a quench occured at the coil \#1. As shown in the upper three curves, the voltages on \#2 and \#3 coils are opposite to that on the coil \#1, which is forced by the warm diode. The gate voltage of the diode at $4.5 \mathrm{~K}$ is measured to be around 10 volts which is the maximum voltage on each coil. The middle three curves show the magnetic fields of each coil which are equivalent to the currents of each coil. The current of the coil \#2 increases due to magnetic induction before its own quenching. This kind of behavior is usually observed when a quench occurs to the coils which are magnetically coupled but electrically independent.

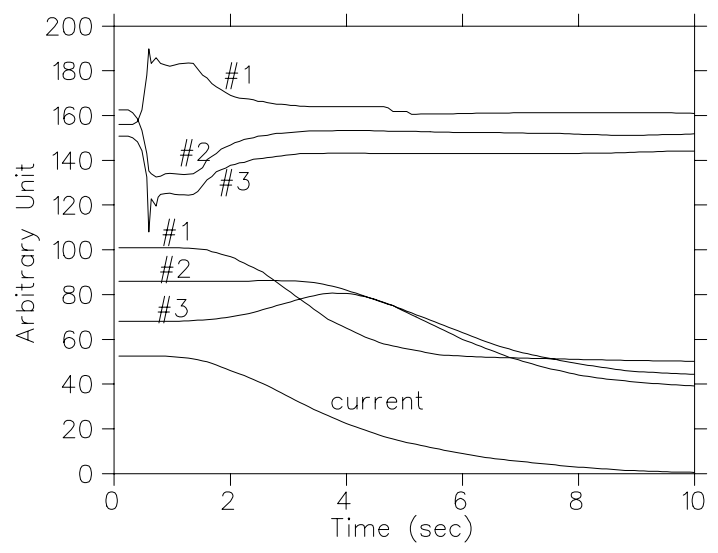

Figure 3: Variations of physical parameters measured as a function of time during coil quench testes. The upper three curves show the coil voltages, the middle three displaying magnetic fields measured at the outside of the vacuum jacket around each coil, and the lower curve shows the decay of the solenoid current.

The magnetic field was measured at the factory site using a hall probe positioned by a three dimensional mover. The magnetic center was found from the measurements, and it agrees with the mechanical center. Magnetic field measured along the axis is shown in Figure 4 together with the calculation result using TOSCA [4].

\section{BEAM FOCUSING TEST}

The main design parameters of the solenoid and its location were chosen to focus heavy ion beams with a spot diameter of $0.4 \mathrm{~mm}$ when beam emittance is $3 \pi \mathrm{mm} \cdot \mathrm{mrad}$. The beam envelopes were calculated using TRANSPORT [5], and shown in Figure 5. The beam envelopes are enlarged by the quadrupoles upstream from the solenoid, and their upper limits are determined by the clear bore size $(\phi 8 \mathrm{~cm})$ of the last quadrupole. Aberrations on the solenoid are not the limiting factors in producing the spot size of $0.4 \mathrm{~mm}$. 


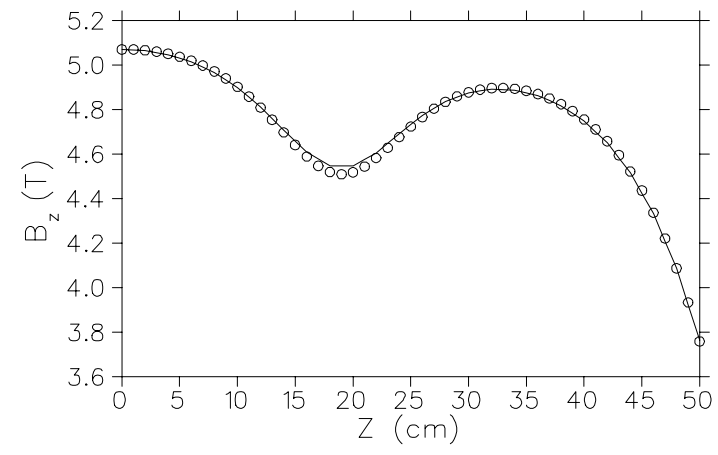

Figure 4: Magnetic fields calculated with TOSCA drawn by the curve and measured with a hall probe indicated by circles

The first beam test was carried out using $95 \mathrm{MeV} / \mathrm{u}$ $\mathrm{Ar}^{17+}$ beam. The spot was observed on a $\mathrm{ZnS}$ target at the location of the production target of radio isotope beams. The beam diameter was around $0.3 \mathrm{~mm}$ when the beamline magnets were adjusted according to the TRANSPORT calculations. The optics calculation agreed with measurements, and the transmission efficiency was near $100 \%$.

The beam spot can be easily shaped with control of three upstream quadrupole doublets as well as solenoid current. With the solenoid inductance of $534 \mathrm{H}$ and the power supply voltage of 4 volts, the adjustment of the solenoid current takes five minutes per amp. Therefore, it is quicker to control the currents of the quadrupoles as long as the transmission efficiency doesn't deteriorate.

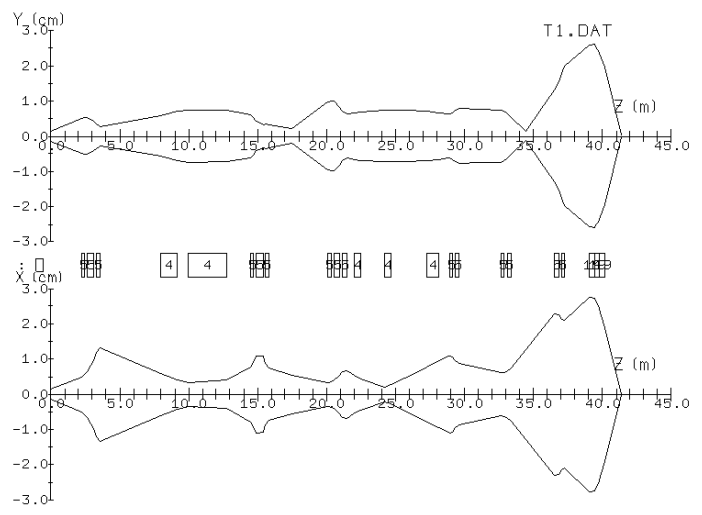

Figure 5: Beam envelopes calculated with TRANSPORT. The upper and lower graphs show the envelopes of two transverse planes, respectively.

\section{CONCLUSION}

The solenoid reached the design field of 6.2 tesla, and the beam test was successfully carried out. The number of quench before reaching the design field was larger than expected, but it was understandable considering training against the changes in coil stress distributions from the individual coil tests.

It appears easy to operate a superconducting magnet using conductive cooling. A further application of this kind of system is under consideration.

\section{REFERENCES}

[1] I. Tanihata, RIKEN Accelerator Research Facility, Nuclear Physics News, V5, No1 (1995) 5

[2] T. Kubo et al., The RIKEN Radioactive Beam Facility, B70 NIM (1992) 309

[3] T. Ogitsu et al., Superconducting focusing solenoid for Xband klystron, Proceedings of 1995 Partl. Accel. Conf. (1995) 1405

[4] Vector Fields Limited, Oxford, England

[5] K. Brown et al., TRANSPORT. A Computer Program for Designing Charged Particle Beam Transport System, CERN 7316 (1993) 